\title{
Mechanisms of Podocyte Detachment, Podocyturia, and Risk of Progression of Glomerulopathies
}

\author{
Hernán Trimarchi
}

Nephrology Service, Department of Internal Medicine, Hospital Británico de Buenos Aires, Buenos Aires, Argentina

\section{Keywords}

Podocyte · Podocyturia · Proteinuria · Glomerulopathy ·

Chronic kidney disease

\begin{abstract}
Background: Glomerulopathies are the main cause of ESRD. Primary or secondary causes of glomerular diseases comprise more than $70 \%$ of cases that end up in renal replacement therapies. Summary: The total glomerular mass that each individual contains is key to maintaining normal kidney function. Diabetes, hypertension, and any primary or secondary glomerulopathy may threaten the normal glomerular function. In fact, any glomerular insult may alter the glomerular filtration barrier, which in turn is composed by the podocyte, the glomerular basement membrane, and the capillary endothelial cell. Deposition of immune complexes, antibodies, or complement components at the subepithelial, intramembranous, or subendothelial space, and mutations in podocyte, slit diaphragm, or glomerular basement membrane proteins or enzymes are the main etiologies of glomerular alterations. Podocytes are glomerular cells that do not divide under normal circumstances. In this respect, maintenance of the absolute podocyte number per glomer-
\end{abstract}

ulus is critical for normal glomerular function. As the insult progresses, podocytes start to detach from the glomerular basement membrane. When the podocyte loss is over $40 \%$ in a glomerulus, glomerulosclerosis develops, and obliteration of the glomerulus is the rule. In clinical grounds, this phenomenon is diagnosed mainly by proteinuria and a decline in glomerular filtration rate. Key messages: In this review article, the impact of podocyturia in glomerular diseases and the main mechanisms of podocyte detachment are discussed. Finally, potential targets of therapeutic approach are suggested.

(c) 2020 The Author(s)

Published by S. Karger AG, Basel

\section{Background}

CKD is associated with an elevated rate of morbidity, kidney failure, the requirement for dialysis or kidney transplantation, and elevated mortality compared to the general population [1]. The worldwide prevalence of CKD is estimated to be approximately between 10 and $15 \%$ and is a major contributor to the costs of health care [2]. Proteinuria and estimated glomerular filtration rate 
(eGFR) are currently the most widely used predictors of CKD prognosis and kidney involvement [3].

Several studies have shown that the assessment of podocyturia may be an effective biomarker of kidney damage, mainly due to glomerular diseases [4-6]. Podocytes are highly differentiated glomerular cells with limited capacity for cell division that form the interdigitating foot processes located on the outer surface of the glomerular capillaries in Bowman's capsule [7]. Podocytes are essential for the permeability of the capillary filtration barrier and prevent the leakage of macromolecules and blood cells to the glomerular filtrate. Progressive detachment of podocytes eventually leads to proteinuria and progressive kidney failure [7-9]. Several unique proteins such as podocalyxin, podocin, nephrin, and synaptopodin are located on podocytes or in their shed extracellular vesicles [10-12]. The effacement of podocyte foot processes and the secondary increased concentrations of podocytes in the urine in patients with glomerular diseases have been shown to correlate with clinical severity and progression and ought to be taken into consideration in order to evaluate the degree of glomerular involvement and of renal function prognosis [12-16].

\section{Assessment of Podocyturia}

Current methodologies for the evaluation of podocyturia in patients are challenging [17-19]. Methods employing immunofluorescence of specific podocyte proteins are based on colorimetric interpretation and, as such, lack of exact quantitative analysis. Moreover, cell counting to evaluate podocyturia is time-consuming, offers a limited sensitivity, is operator-dependent, and is also expensive owing to the need to use specific antibodies $[17,18]$. The quantitative analysis of mRNAs by qPCR has been used to quantify specific podocyte protein mRNAs in urine and in podocytes as well $[20,21]$, but the quality is limited, leading to increased variability $[22,23]$. Liquid chromatography coupled to tandem mass spectrometry was also developed [24-26]. However, as podocyturia has not been validated, it should not be employed in clinical grounds. It can be used as an additional tool to assess the degree of glomerular damage at early or subclinical stages of glomerular diseases, as it detects glomerular involvement even before proteinuria ensues. Moreover, the diagnosis of podocyturia is not only related to the method employed and to the skill of the operator but also to the tagged podocyte protein. Subpopulations of podocytes may be encountered at different stages of the same glomerular disease or in different glomerulopathies $[27,28]$.

\section{Podocyte and the Glomerulus}

The podocyte is a highly differentiated cell phylogenetically mainly devoted to avoid the loss of the huge amount of proteins delivered to the kidney every day [7, 8]. As an example, if we consider an average normal serum albumin concentration of $4.5 \mathrm{~g} / \mathrm{dL}$, a daily filtered blood volume of $200 \mathrm{~L}$ faces the glomerular mass with $9 \mathrm{~kg}$ of albumin. However, none or minute amounts of albumin are normally excreted in the urine. The successful task podocytes perform may explain why these cells have been highly preserved along life on Earth. Podocytes also intervene in many other important functions that turn the glomerular apparatus an efficient structure. Podocytes secrete components of the glomerular basement membrane, as vascular endothelial growth factor, a molecule that maintains glomerular endothelial fenestrae patent and open, to augment the filtration surface. Podocytes also interact with the immune system, as antigenpresenting cells, as receptors of complement components and of immunoglobulins $[10,17]$. Their delicate and precise foot process interaction with the slit diaphragm, basement membrane, and adjacent endothelial cells determines the characteristics of the protein-devoid glomerular ultrafiltrate. However, their location in the outer space of the glomerular filtration barrier and lying against the constant flow of the filtrate may be hypotheses to explain the reason why they are unable to divide under normal circumstances $[7,8,10,17]$. As mentioned above, podocytes can undergo karyokinesis but not cytokinesis. Interestingly, in an individual, the highest amount of podocytes is reached at birth, slowly declining along the decades $[5,14]$. Therefore, it is critical to maintain the normal mass of podocytes, in order to avoid loss of proteins in the urine. It is well known that proteinuria is a marker of progression of kidney disease and is also a risk factor for cardiovascular disease [3, 29].

In the last 20 years, over 50 genetic mutations leading to deletions or structural modifications of podocyte proteins have been reported as important causes of podocytopathies and of secondary causes of focal and segmental glomerulosclerosis. Besides genetic factors, podocyte dysregulation and injury leading to proteinuria may also be due to still not yet identified permeability factor/s, likely active in minimal change disease or primary focal seg- 
mental glomerular sclerosis (FSGS, leading to the modification of the glomerular barrier permselectivity in the absence of any inflammatory changes [22]. Podocytes can also react through specific receptors to a variety of inflammatory mediators, including cytokines, reactive oxygen species, and complement products released during glomerular inflammatory conditions [30].

Podocytes can suffer from hemodynamic challenges, due to their downstream localization from the glomerular basement membrane, which expose these cells to constant stress, further amplified in hyperfiltration conditions $[8,30]$. In addition, hyperfiltration due to a reduced number of functioning nephrons can also induce hemodynamic and architectural podocytopathic changes, which can be detected in both primary and secondary forms of FSGS. In these various conditions, podocyte activation by immunological and/or hemodynamic factors triggers a cascade of protein kinase activity leading to reactive oxygen species release, endoplasmic reticulum stress, protein unfolding abnormalities, and mitochondrial damage. The podocyte stress burden impacts the integrity of the cytoskeleton and slit diaphragm, promoting cell functional and anatomic damage, detachment, and death $[8,10,14,31]$.

It is of interest for understanding the podocyte damage in conditions with expansion of the mesangial area, as it occurs in IgA nephropathy (IgAN), to consider the functional relationship between glomerular capillaries and the folding pattern of the glomerular basement membrane [32]. The peripheral outpocketings of the glomerular basement membrane form a continuous channel system that is open to the mesangial area and contains the capillaries. The mechanical stability of this system is largely maintained by the mesangial cells, which insert along the paramesangial aspect of the glomerular basement membrane. In conditions of increased mesangial area, there is a lengthening of the mesangial axis, followed by the prolapse of the capillary and the associated podocyte detachment with loss into the urinary space $[32,33]$. Thus, the podocyte stress and adaptation observed in podocytopathies manifest as alterations in podocyte charge or shape, an active process that occurs due to actin cytoskeleton rearrangement that precedes podocyturia $[8,13,30]$.

\section{Podocyturia and Glomerular Diseases}

In glomerular diseases, any insult directed to the endothelial cell, to the glomerular basement membrane, and/or to the podocyte itself will determine a disruption in the proper function of the glomerular filtration barrier. With respect to the podocyte, the consequences of this derangement will cause an adaptation of the cell to remain in place at any cost. Morphologically, this is observed as podocyte foot process effacements, which are associated with proteinuria and hyperfiltration at the initial phases. Moreover, when the situation is not corrected or balanced, then podocytes start to detach, podocyturia ensues, proteinuria increases, and glomerular filtration rate decreases $[8,14,23]$. This advanced and irreversible situation is histologically reported as glomerulosclerosis, with tuft adherences and different degrees of mesangial expansion. It is believed that when over $40 \%$ of the podocyte population per glomerulus is lost, with focal and segmental glomerulosclerosis as the main picture of the kidney biopsy, the glomerulus starts a "point of no return," and glomerular obliteration is the irreversible result [14].

Primary FSGS is defined as a podocytopathy since podocyte injury with cytoskeleton disorganization and foot process effacement are the initiating mechanisms leading to loss of adaptation and detachment from the glomerular basement membrane. Large podocyte loss results in focal adherence to parietal epithelial cells and scarring. At this point, an absolute $40-50 \%$ podocyte loss per glomerulus is supposed to have occurred $[10,14]$. As a result, activated parietal epithelial cells start to synthetize extracellular matrix and then migrate to a segment of the glomerular tuft where denudated podocyte-depleted glomerular basement membrane exists. It is from this site that podocytes are detached, the matrix is deposited, and FSGS develops [24].

In the spectrum of glomerular diseases, it has been demonstrated that podocyte detachment is the mechanism by which proteinuria appears and glomerular filtration rate decreases. We have demonstrated that one of the mechanisms by which podocytes detach is when the urokinase-type plasminogen activator receptor (uPAR), a multi-domain glycoprotein which normally binds to urokinase plasminogen activator, which is located at the basal site of the podocyte, and also couples with integrins $\alpha \mathrm{V} \beta 3$ or $\alpha 3 \beta 1$, which are responsible for the attachment of the cell to the basement membrane $[23,25,26]$. Briefly, integrins are transmembrane receptors for various extracellular membrane components, including laminin, vitronectin, collagens, and fibronectin. They activate kinases that phosphorylate key molecules for cell-cell and cellmatrix interactions $[25,26]$. Thence, the mentioned interaction causes the engulfment of the uPAR-integrin complex by the podocyte, with the subsequent intracel- 
lular contraction of actin filaments. For instance, the binding of uPAR to podocyte $\alpha \mathrm{V} \beta 3$ induces foot process effacement and eventually podocyte detachment $[23,25$, 26]. In the experimental model of LPS-induced nephropathy, UPAR is the key factor for mediating foot process effacement and proteinuria by interaction with $\alpha \mathrm{V} \beta 3$, leading to integrin activation and podocyte damage [26, 34].

It is this persistent stimulation of the contractile cytoplasmic apparatus, the main cause of podocyte stress, that determines the detachment of the cell. Therefore, the denuded glomerular basement membrane is covered by adjacent podocytes in order to avoid the loss of proteins in the urine. When the absolute number of podocytes is decreased enough, the basement membrane becomes denuded permanently, proteinuria becomes a permanent finding, and glomerulosclerosis appears as a chronic irreversible feature $[10,14,31]$.

Another relevant integrin member playing a role in podocyte survival and involved in acquired damage is a $3 \beta 1$, which binds with its intracellular domain cytoskeletal actin and with the extracellular domain fibronectin, collagen, and laminin, reacting also with activated $\mathrm{T}$ cells via CD80 (B7-1), a costimulatory receptor on antigenpresenting cells. This molecule was found to be induced in experimental models of glomerular diseases with proteinuria, following the activation of innate immune signaling via TLR- 4 by bacterial endotoxin (LPS) [34]. CD80 modifies the actin cytoskeleton of podocytes and modulates slit diaphragm disposition. Addition of TNFa to cultured podocytes causes CD80 upregulation, actin reorganization, and podocyte injury [35]. In humans, CD80 was upregulated in several proteinuric states, including primary FSGS [36]. Under podocyte stress conditions, the increased expression of CD80 on the podocyte surface leads to high urinary CD80 excretion, as detected in different proteinuric conditions $[37,38]$.

This podocyte loss is accompanied by an important inflammatory response, which initially does not imply the affluence or inflammatory cells in kidney biopsies. In this regard, B7-1/CD80 is also a marker present from the initial phases of the inflammatory process of certain glomerulopathies with necessarily no cellular inflammatory cell presence in a kidney biopsy [39]. Noteworthy, under abnormal conditions, there is an upregulation of CD80 expression on podocyte surfaces, suggesting that these cells may functionally turn into antigen-presenting-like cells. We have reported that CD80 urinary excretion is significantly increased in patients with Fabry disease as in those patients not consid-

Podocyturia and Glomerular Diseases ered to be under therapy due to the lack of renal or extrarenal compromise [37]. However, Fabry disease is not generally considered a classical inflammatory entity, at least from the histological point of view. This finding suggests that inflammation is present from early stages of the disease at the molecular level, without the histological correlation of the presence of inflammatory cells. We have recently shown a higher degree of proteinuria in patients with IgAN and S1 (segmental glomerulosclerosis) in addition to M1 (mesangial proliferation) than in those with M1 but without segmental sclerosis. Patients with S1 had a more rapid decline in the eGFR and more persistent proteinuria and podocyturia after 7.5 years of follow-up [38]. Finally, we have reported in a pilot study that in stable conditions, analyzed urinary samples of patients with IgAN (unchanged eGFR and proteinuria persistently $<0.75 \mathrm{~g} /$ day in the last 12 months) presented the highest rates of urinary podocyte loss compared to other primary or secondary glomerulopathies or controls [15].

There are several histological hints that may suggest that podocytes are under stress, with their cytoskeleton contracted in a stage prior to detachment. Podocyte hypertrophy, as observed in certain cases of glomerulosclerosis, IgA nephropathy, and chronic diabetic kidney disease, may be an example of this statement [40-42]. Podocyte engorgement and slit diaphragm widening due to the existence of cytoplasmic reabsorbed proteins or the accumulation of mutated enzyme substrates, as GB3 in the case of Fabry nephropathy, may be another one $[43,44]$. Primary endothelial swelling has also been considered as a hallmark of severe entities such as preeclampsia, in which podocyturia has been well characterized. The elevated loss of podocytes in preeclampsia may be secondary to the initial aggression to the glomerular endothelium $[16,45]$. We and others have also documented an elevated podocyturia count in Alport's disease, where a mutation in glomerular basement membrane collagen IV determines a relentless and progressive malfunction of the glomerular filtration barrier [46]. In proliferative glomerulonephritis, as extracapillary glomerulonephritis or membranoproliferative classes of lupus nephritis, podocyturia is significantly increased compared to non-progressors, as minimal change disease [5]. However, as mentioned previously, in non-proliferative primary or secondary cases due to genetic causes of focal and segmental glomerulosclerosis, podocyturia is also elevated $[6,46]$.

Interestingly, podocytes are not always damaged at the same cellular compartment level. In Fabry nephropathy, 
we have observed that the number of podocalyxin-positive urinary podocytes is decreased compared to the total amount of these cells. This observation was present in all patients, but significantly more pronounced in advanced stages of the disease. In addition, we have reported that the loss of podocalyxin may also be involved in podocyte loss [27]. Therefore, these findings may not only suggest that the glycocalyx is damaged early in Fabry nephropathy but also indicate that the assessment of podocyturia cannot depend on the tagging of a single podocyte protein. I believe that a thorough assessment of podocyturia could be better accomplished by the identification of proteins belonging to at least 2 different cellular compartments, including the glycocalyx, the cytoplasm, the basal side, and the foot process that lies contiguous to the slit diaphragm.

\section{Potential Therapeutic Implications}

The pharmacological intervention to prevent or decrease the detachment of podocytes from the glomerular basement membrane could avoid or diminish the development of glomerulosclerosis. Amiloride may be an option, since this drug inhibits the synthesis of uPAR at the mRNA level [47]. As a consequence of this inhibition, the activation of $\alpha \mathrm{V} \beta 3$ integrins would be dampened and podocyte detachment decreased $[46,48,49]$. The CD80nephrin coupling is another potential therapeutic target [35]. Abatacept (which interferes with the lymphocyte CD28-antigen-presenting cell CD80 coupling) has been reported to be associated with the development of IgAN. Thus, CD80 emerges as another potential target to assess in the therapy of IgAN [39].

\section{Limitations}

For the present time, podocyturia should be destined to contribute to unraveling the pathophysiological pathways of glomerular diseases. It is not a validated method to be employed in clinical grounds. Moreover, it is timeconsuming, expensive, and must be performed by welltrained professionals.

\section{Conclusion}

Podocytes are highly specialized cells located in the glomeruli, are incapable of undergoing mitosis under normal conditions, and are mainly committed to avoid the loss of proteins in the urine. Their loss in the urine, defined as podocyturia, determines the appearance of glomerulosclerosis, proteinuria, and CKD. Podocyturia antedates proteinuria as a biomarker of kidney dysfunction, but at the present time, it is not a validated method to be employed in clinical grounds. Its main indication is to unravel the pathophysiological mechanisms of glomerular diseases.

\section{Disclosure Statement}

The author has no conflicts of interest to declare.

\section{Funding Sources}

The author did not receive any funding.

\section{Author Contribution}

The author has written the whole manuscript himself.

\section{References}

1 Levin A, Tonelli M, Bonventre J, Coresh J, Donner JA, Fogo AB, et al. Global Kidney Health 2017 and beyond: a roadmap for closing gaps in care, research, and policy. Lancet. 2017;390(10105):1888-917.

2 Hill NR, Fatoba ST, Oke JL, Hirst JA, O'Callaghan CA, Lasserson DS, et al. Global prevalence of chronic kidney disease: a systematic review and meta-analysis. PLoS One. 2016;11(7):e0158765.

3 Nash DM, Brimble S, Markle-Reid M, McArthur E, Tu K, Nesrallah GE, et al. Quality of care for patients with chronic kidney disease in the primary care setting: a retrospective cohort study from Ontario, Canada. Can J Kidney Health Dis. 2017 Apr 11;4:2054358117703059.
4 Vogelmann SU, Nelson WJ, Myers BD, Lemley KV. Urinary excretion of viable podocytes in health and renal disease. Am J Physiol Renal Physiol. 2003;285(1):F40-8.

5 Wickman L, Afshinnia F, Wang SQ, Yang Y, Wang F, Chowdhury M, et al. Urine podocyte mRNAs, proteinuria, and progression in human glomerular diseases. J Am Soc Nephrol. 2013;24(12):2081-95.

6 Trimarchi H, Canzonieri R, Muryan A, Schiel A, Araoz A, Forrester M, et al. Copious podocyturia without proteinuria and with normal renal function in a young adult with Fabry disease. Case Rep Nephrol. 2015;2015:257628.

7 Jefferson JA, Alpers CE, Shankland SJ. Podocyte biology for the bedside. Am J Kidney Dis. 2011;58(5):835-45.
8 Kriz W, Shirato I, Nagata M, LeHir M, Lemley KV. The podocyte's response to stress: the enigma of foot process effacement. Am J Physiol Renal Physiol. 2013;304(4):F333-47.

9 Jefferson JA, Nelson PJ, Najafian B, Shankland SJ. Podocyte disorders: core curriculum 2011. Am J Kidney Dis. 2011;58(4):666-77.

10 Trimarchi H. Podocyturia: what is in a name? J Transl Int Med. 2015;3(2):51-6.

11 Craici IM, Wagner SJ, Bailey KR, Fitz-Gibbon PD, Wood-Wentz CM, Turner ST, et al. Podocyturia predates proteinuria and clinical features of preeclampsia: longitudinal prospective study. Hypertension. 2013;61(6): 1289-96. 
12 Fall B, Scott CR, Mauer M, Shankland S, Pippin J, Jefferson JA, et al. Urinary podocyte loss is increased in patients with Fabry disease and correlates with clinical severity of Fabry nephropathy. PLoS One. 2016;11(12):e0168346.

13 Trimarchi H, Canzonieri R, Schiel A, Politei J, Stern A, Andrews J, et al. Erratum to: podocyturia is significantly elevated in untreated vs treated Fabry adult patients. J Nephrol. 2016; 29(6):791-7.

14 Wiggins RC. The spectrum of podocytopathies: a unifying view of glomerular diseases. Kidney Int. 2007;71(12):1205-14.

15 Trimarchi H, Canzonieri R, Muryan A, Schiel A, Iotti A, Aráoz A, et al. La identificación de podocitos urinarios. la utilidad potential de un biomarcador novedoso de daño glomerular en las glomerulopatías primarias y secundarias. Un estudio piloto. Nefrología Argentina. 2015;13:85-92.

16 Martineau T, Boutin M, Côté AM, Maranda B, Bichet DG, Auray-Blais C. Tandem mass spectrometry analysis of urinary podocalyxin and podocin in the investigation of podocyturia in women with preeclampsia and Fabry disease patients. Clin Chim Acta. 2019;495 67-75.

17 Trimarchi H. Podocyturia: potential applications and current limitations. World J Nephrol. 2017;6(5):221-8.

18 Puelles VG, Bertram JF, Moeller MJ. Quantifying podocyte depletion: theoretical and practical considerations. Cell Tissue Res. 2017;369(1):229-36.

19 Hagmann H, Brinkkoetter PT. Experimental models to study podocyte biology: stock-taking the toolbox of glomerular research. Front Pediatr. 2018;6(193):193-9.

20 Kelder TP, Penning ME, Uh HW, Cohen D, Bloemenkamp KW, Bruijn JA, et al. Quantitative polymerase chain reaction-based analysis of podocyturia is a feasible diagnostic tool in preeclampsia. Hypertension. 2012;60(6) 1538-44.

21 Furuta I, Zhai T, Umazume T, Ishikawa S, Hosokawa A, Kojima T, et al. Post-partum podocyturia following pre-eclamptic pregnancy. J Obstet Gynaecol Res. 2017;43(6) 1008-13.

22 Le Berre L, Godfrin Y, Lafond-Puyet L, Perretto S, Le Carrer D, Bouhours JF, et al . Effect of plasma fractions from patients with focal and segmental glomerulosclerosis on rat proteinuria. Kidney Int. 2000;58(6):2502-11.

23 Trimarchi H. Primary focal and segmental glomerulosclerosis and suPAR: where do we stand and where are we heading to? World J Nephrol. 2013;2:103-10.

24 Ponticelli CE, Glassock RJ, Scolari F. Treatment of focal segmental glomerulosclerosis. In: Ponticelli C, Glassock RJ, editors. Treatment of primary glomerulonephritis. 3 rd ed. Chapter 5. Oxford: Oxford University Press 2019. p. 259-46.
25 Wei Y, Eble JA, Wang Z, Kreidberg JA, Chapman HA. Urokinase receptors promote beta 1 integrin function through interactions with integrin $\alpha 3 \beta 1$. Mol Biol Cell. 2001;12(10): 2975-86.

26 Wei C, Möller CC, Altintas MM, Li J, Schwarz $\mathrm{K}$, Zacchigna S, et al. Modification of kidney barrier function by the urokinase receptor. Nat Med. 2008;14(1):55-63

27 Trimarchi H, Canzonieri R, Costales-Collaguazo C, Politei J, Stern A, Paulero M, et al. Early decrease in the podocalyxin to synaptopodin ratio in urinary Fabry podocytes. Clin Kidney J. 2019;12(1):53-60.

28 Maestroni S, Maestroni A, Dell'Antonio G Gabellini D, Terzi S, Spinello A, et al. Viable podocyturia in healthy individuals: implications for podocytopathies. Am J Kidney Dis. 2014;64(6):1003-5.

29 Rovin BH, Caster DJ, Cattran DC, Gibson KL, Hogan JJ, Moeller MJ, et al. Management and treatment of glomerular diseases (part 2): conclusions from a kidney disease: improving global outcomes (KDIGO) controversies conference. Kidney Int. 2019;95(2):281-95.

30 Nagata M. Podocyte injury and its consequences. Kidney Int. 2016;89(6):1221-30.

31 Wharram BL, Goyal M, Wiggins JE, Sanden SK, Hussain S, Filipiak WE, et al. Podocyte depletion causes glomerulosclerosis: diphtheria toxin-induced podocyte depletion in rats expressing human diphtheria toxin receptor transgene. J Am Soc Nephrol. 2005;16(10): 2941-52.

32 Kriz W. Maintenance and breakdown of glomerular tuft architecture. J Am Soc Nephrol. 2018;29(4):1075-7.

33 Trimarchi H, Coppo R. Podocytopathy in the mesangial proliferative immunoglobulin $\mathrm{A}$ nephropathy: new insights into the mechanisms of damage and progression. Nephrol Dial Transplant. 2019;34(8):1280-5.

34 Reiser J, von Gersdorff G, Loos M, Oh J, Asanuma K, Giardino L, et al. Induction of B7-1 in podocytes is associated with nephrotic syndrome. J Clin Invest. 2004;113(10):1390-7.

35 Khullar B, Balyan R, Oswal N, Jain N, Sharma A, Abdin MZ, et al. Interaction of CD80 with Neph1: a potential mechanism of podocyte injury. Clin Exp Nephrol. 2018;22:508-16.

36 Cara-Fuentes G, Wei C, Segarra A, Ishimoto T, Rivard C, Johnson RJ, et al. CD80 and suPAR in patients with minimal change disease and focal segmental glomerulosclerosis: diagnostic and pathogenic significance. Pediatr Nephrol. 2014;29(8):1363-71.

37 Trimarchi H, Canzonieri R, Schiel A, Costales-Collaguazo C, Politei J, Stern A, et al. Increased urinary CD80 excretion and podocyturia in Fabry disease. J Transl Med. 2016; 14(1):289-8.
38 Trimarchi H, Canzonieri R, Schiel A, Costales-Collaguazo C, Stern A, Paulero M, et al. In IgA nephropathy, glomerulosclerosis is associated with increased urinary CD80 excretion and urokinase-type plasminogen activator receptor-positive podocyturia. Nephron Extra. 2017;7(2):52-61.

39 Trimarchi H. Abatacept and glomerular diseases: the open road for the second signal as a new target is settled down. Recent Pat Endocr Metab Immune Drug Discov. 2015;9(1):2-14.

40 D'Agati VD, Fogo AB, Brujin JA, Jennette JC. Pathologic classification of focal segmental glomerulosclerosis: a working proposal. Am J Kidney Dis. 2004;43:368-82.

41 Bellur SS, Lepeytre F, Vorobyeva O, Troyanov S, Cook HT, Roberts IS. Evidence from the Oxford Classification cohort supports the clinical value of subclassification of focal segmental glomerulosclerosis in IgA nephropathy. Kidney Int. 2017;91(1):235-43.

42 Miyauchi M, Toyoda M, Kobayashi K, Abe M, Kobayashi T, Kato M, et al. Hypertrophy and loss of podocytes in diabetic nephropathy. Intern Med. 2009;48(18):1615-20.

43 Tøndel C, Kanai T, Larsen KK, Ito S, Politei JM, Warnock DG, et al. Foot process effacement is an early marker of nephropathy in young classic Fabry patients without albuminuria. Nephron. 2015;129(1):16-21.

44 Trimarchi H, Karl A, Raña MS, Forrester M, Pomeranz V, Lombi F, et al. Initially nondiagnosed Fabry's disease when electron microscopy is lacking: the continuing story of focal and segmental glomerulosclerosis. Case Rep Nephrol Urol. 2013;3(1):51-7.

45 Jim B, Jean-Louis P, Qipo A, Garry D, Mian $\mathrm{S}$, Matos T, et al. Podocyturia as a diagnostic marker for preeclampsia amongst high-risk pregnant patients. J Pregnancy. 2012;2012: 984630.

46 Trimarchi H, Canzonieri R, Muryan A, Schiel A, Araoz A, Paulero M, et al. Podocyturia: a clue for the rational use of amiloride in Alport renal disease. Case Rep Nephrol. 2016;2016: 1492743.

47 Zhang B, Xie S, Shi W, Yang Y. Amiloride offtarget effect inhibits podocyte urokinase receptor expression and reduces proteinuria. Nephrol Dial Transplant. 2012;27(5):174655.

48 Trimarchi H, Forrester M, Lombi F, Pomeranz V, Raña MS, Karl A, et al. Amiloride as an alternate adjuvant antiproteinuric agent in Fabry disease: the potential roles of plasmin and uPAR. Case Rep Nephrol. 2014;2014: 854521.

49 Trimarchi H, Paulero M, Canzonieri R, Schiel A, Iotti A, Costales-Collaguazo $\mathrm{C}$, et al. In acute IgA nephropathy, proteinuria and creatinine are in the spot, but podocyturia operates in silence: any place for amiloride? Case Rep Nephrol. 2017:2017:1292531. 